595.7
$k 45 n$

lerner 

(9)

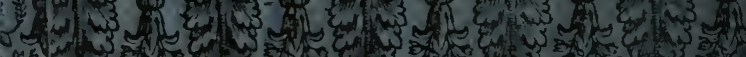

1.
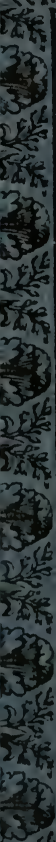

$5 \sqrt{5}$
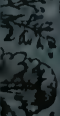

Sis

2tis

3

5

Eर्स:

33

3.3

25

(1)

$B^{2} \mathrm{I}$

S

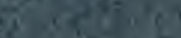




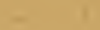





\section{Paturgeffictite Der}

Coccus Bromelia oder des

\section{Ina a a bithilldeb.}

\section{debfit eillem}

auf Erfohtrung gegrůndeten כorfchlag зur gámblicben ßertilgung

Difez

\section{oer 2luanaspflange}

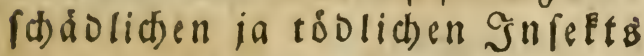
wott

Э. S. Rerner

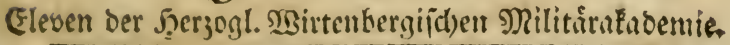

init einer zippertafel.

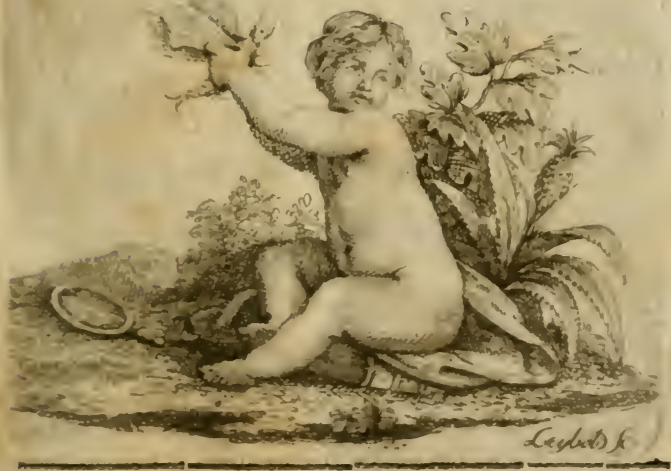

Stuttgart

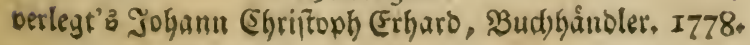




$$
* * * * * * *
$$

Rerum natura nusquam magis, quam in minimis, tota eft.

Plin. hift.nat. Lib. XI, c, 2. 


\section{(2)}

Sano zu geben, wie baffelbige auf Das fchil: lichfere vertilget werben fan.

Die Erfafrungen, auf bie idf micf)

ftuse, find von mir oft, genau und forgs fältig angeftellt, uno befonders von einem Mann mefrmalen wiberfolet worden, Der fein ganges Seben mit phyfifalifden 2 Sabses nefmungen sugebractit bat.

Şei allen meinen $23 e 0 b a c t$ tungen babe ith) niemals entbefen fơnnen: Daß Das ฏåann: then ber Bromelia mit Slugeln ausgeruffet feye. 2tuch Die Coccus Aonidum, (*) welche

(*) Bilašbeeterfifillo. Die Coccus Polonicus, polnif(d)e ßirneridoillo. 


\section{(2)}

$\sqrt{2})^{24}$

weldfe Der Siitter won Limme befefreibet, hat

Feine Slúgel. Der Siitter bátte mithin bie

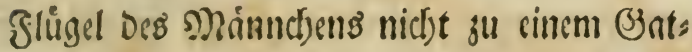
tungsmerfmal mactien follen. Willeicht lernt-man oumet) neuere Entoctungen nod) meforere Strten Fennen, Die Der Schipfer nicht mit Sluggeln liegabet bat.

Dic :latut thut nichts umfonft. Sci allen Sticrarten ftefen Die Empfinoungs's und Serwegungstwerfiscuge, Eurs, Der ganje Eorperliche Sau, in ber genaueften llebercins ftimmung mit Den Eorperliclen Derrictstuns sen, oder mit Der 2jeftinmung Dis ₹̧hieres. Difí ift oer Sall bei ocr Bromelia. Der ) 3 Dit 
Srt iffes 2ufenthaltes, Die 2 ant, wie fie fich nábret uno fortsplanset, macten ithe bie Slú: gel entbefrlict. Urfactse genug, rarum ibe Die 2Ratur feine gegcben bat.

WBenn Beobactitungen, weldfe unter andern Scimmelsftricten, bie entweder waiks mer doer fälter fino, angeftellet roeroen, son Den meinigen bie uno da verfectieden aus̆fals len follten, fo Fan Diß Dennoch Éeinen Bruno gegen Die Siichtigfeit uno Zuberláfigéeit Der meinigen, abgeben.

Uebrigens werde ict) gegrủndete Erinnes rungen gerne uno mit Dane annefmen, aucb meine geringe Arbeit fur genug belohnet bals 


\section{管}

ten, wenn andere baburd) nufgemuntert wers Den, eigene S3eobact)tungen ansuftellen, um Daburch) Die meinigen su beftátigen, ober noch meljecrs zu berictitigen. Schriebs in Stutts sart, Den 3 Iten Senner 1778.

\section{Э. ธ. ณ.}

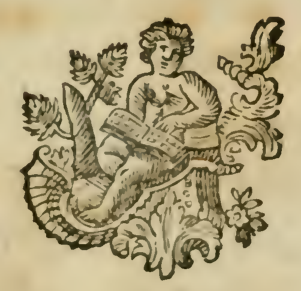

Inthalt. 


\section{Jingalt.}

Einleitung.

S. I.

I. Rapitel. Natuirliche (Scjd)ichte difez Sัniekt

ธ. 5 .

II. Rapitel. Sharafteriftifde 2̧ejdreibung Deffelben.

ธ. 13 .

III. Rapitel. Mittel Dagegen.

ธ. 39 .

Erfter Berfuds.

S.4I.

3 weiter $\mathfrak{B e r j u c t . ~}$

S. 42 .

Dritter $\mathfrak{B e r}$ [ud).

‥ 43 .

Bierter જ̧erjucy.

๔. 45 .

รัubang.

S. 5 I.

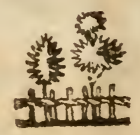

Bins 


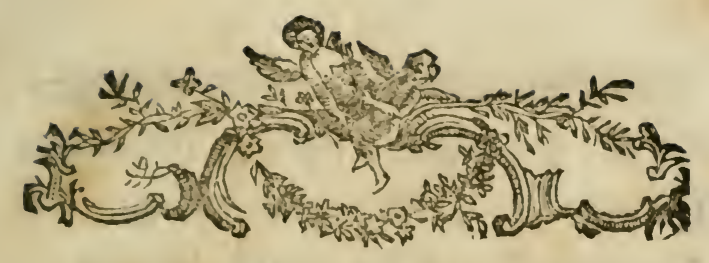

\section{Einleitung.}

S) ie Edwwirigkeit, bas jufeft, mit bef. fen Unterfutfung id) mids) bier bes fd)aftige, in feinen (Eigenfdaften zu burdifor. fdjen, und bie Eeltenţeit ber Beobnditun. gen, bie big̈ger von andern bariber gemadje worben, forberte von mir eine gennuere uno meitlauftigere Sefdrcibung ocffelbigen, als in jeocm anbern fall nơtbig gewefen wäre.

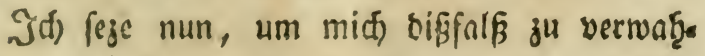
ren, in Znfeţung bes erf̣ten Dunf́ts einige Betrad)eungen voraus. 
Die Edfwirigfeiten, Das Jnfeft, bas ith Fier befdreibe, zu betrad)een, fino fef̧r großs. Rit blofen 2lugen fan man es bei naţe gar nidf) fetcen, und iff alfo genotţ̧iget, bas Bers groferungeglas z" gebraucfen. Allleine, folbft

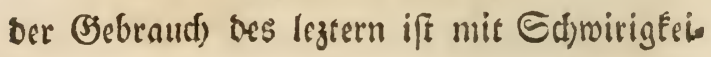
ten verbunden.

Eo balo man es unter bas zufammenges fezte Bergrooferungeglas bringt, (uno diß ift zu einer genauen $\mathfrak{B}$ cobadjung notf̧wendig, )

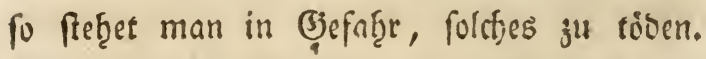
Meberoifes entrifotst das unter bas Dbjef́tiv. glas gebradise Junfeft immer bem 2lug, und man muß baf̧ero entweder bas (S)las ḩin und bzer berwegen, um bas Jnfofte nicht nus bem Eefrefelo zu verlicḩren, oocr man mus es mit einem f̧airchen, fo oft es bayon laufen

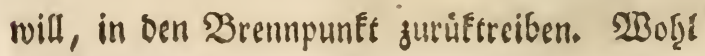


znanjig 2erfude miflungen mir um oicfes Llmfranos willen, sţe ith die Enugruifel uno sie 2lugen entosfen fonnte.

Der Unterfdjeo des Jnfefts bermef̧ret nod) Die Ed)wirigfeit. Eo fan \%. (ङ) ein 2(nanasfdils in einer Beit von vict bis funf Sagen cine gewiffe (5röfe erb̧alten, weld)e bie meiften andern in eben ber Beit nidger reid)en, nod) reniget libertreffen.

Dhtue nod) anoere, bels allell Jinfeften überţaupt gewif̨nliche Edfwirigfeiten aufgu. fudsen, frige id) bier eine 2umerfung bei, Die zugleid) eine (Entfduloigung bes Berfaf. fers ift, im fall bic vorbere gemadjte \$eob. ad)tungen bei gleidjen lluterfudfungen bon an. bern nid)t überall beftitiget wuirben.

(Fs gefdief̧et nemlid) feb̨r oft: Oaß́ ein Znanasfdilo sinige, voll andern gally unter.

$$
\text { 24 } 2
$$

foise 
fdjebene Erfdjeinungen, நृat; eine Berfdjie: Denf̧eit, sie blok von ben unterffoiedenen àfferliden Lumftanden, in benen fid) beibe Exhilbe befanden, நृerrüb̨ret.

Man Ean alfo villeid)t oft nidft bei cinem folchen basjenige antreffen, was ber Berfaffer bei andern, anderswo aufgemad)fenen Ectjilben, gefunden b̧at.

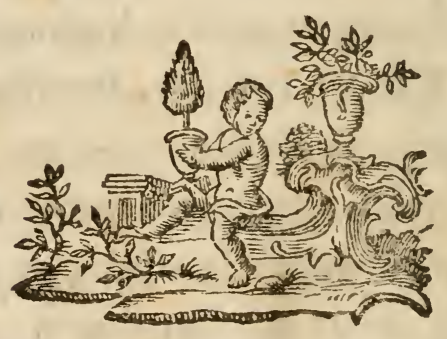

I. 2in 
I. Kinpitel.

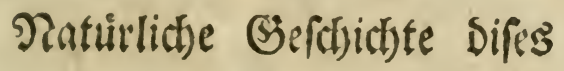

Snretts.

(5) as erfee, was uns bei ber unterfucfung Der (Jeffticifte der Bromelia in oie 2lugen fálte, ift tas Ei, ber Uriprung bes ₹̧̧ierdjens.

I. Utrpruinglid ligen alle (Eier, bie fidf auf ợngeferger 30 bis 50 erfrefen, zerftreutet, untere cinem weiffen Defel in einer $\mathfrak{S}_{\text {gio }}$ lung, uno jebes ift mit einem bủnnen weiffen รุăuttffen überjogen.

II. Z(d)t bis zeŗen Enge nad) if̧rer entffes f̧ung, (bie id) aus ben bamit verbun.

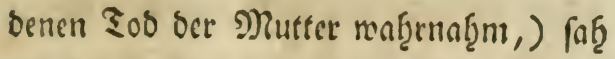
id) fie, bem Edfeine nad), unverainbert. Bierzeģen Sage darauff fand idf bas Ei

$$
\mathfrak{A l}_{3} \text { aufo }
$$


aufgefprungetr, uno das Sgierdien von Demfelben abgelófet, bas jejt, dem Gian. gen nach), (cuaf) noch niche in oenen sin. zelen Tgeilen,) friftbar roar, weil Das (Fi nod) mit ocmfolben zufammentieng.

S6 mun gleidg bas Jnfeft fáon im (Fi mugte gerwad) fen fern, (oenn bas $\mathfrak{W}$ nd) Etfum war bie Utfacbe, bie bas (Ei geofnet feat,) fo war bod) fein Wadisticum, nadjoem es fid) einmal vom Ei abgelópet ḩatte, viel fefneller uno merflider. Zuoor fab̧e man wob̨l cinen Unterfatied des Cies uno des Thierdens; aber man beubachtete nod) Eeinen einzelen Şreil. Man Eonnte fo gar nod) nid)t bie geringfte

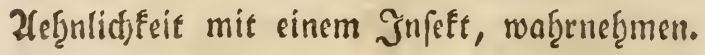

III. Tach verfloffenen adde Sagen fah id) fünf ringe am Seintertheil des rumpfs, 
uns bie Ecţale, in weld)e bns Ţ̧ier. den efremals cingewiest mar, lag, ganj getrent von temlelbigen, am En. De bes Defels.

IV. Sis zum fünf̧igften ₹ag naţnt id nod) feine nerflictje ßerånoctung waḩr, auffer, safj jene Siings immer fidftbarer wurben, uno bas Şierdjen etwas wea niget uudf)s. Die mir zuerft erfdiene. ne Siinge waren mun jejt faft gar nidje meţr fidtthar, weil fie ganj mit f̧aa ren befezt waren.

Iluffer bifem faf̧ man aud am f̧inter. theil zruel) furge farfe Scaare, bie in form ciner Babel auffaffen. 2lü ber 21nfaz zu 2lugen uno Edinnaugen, ober Saugrüfel, wa.

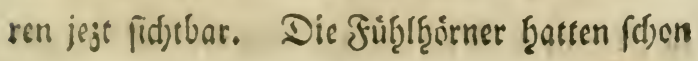

$$
\mathfrak{X}_{4} \text { oen }
$$


Den vierten Theil bes ganjen Rörpers in oer Sange; fie endigten fid in sine fefer frine Epize, uno if̧re Serveglidbereit mar gar nicht mef̨r nuffer Znocifel zu fezen. Heber.

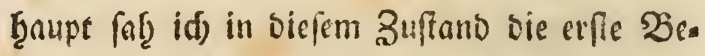
wegung bes ganzen Jufefts.

Nacf) zroanjig Tagen weiter fallo idf bas

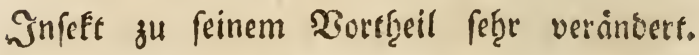
zllle Theile waren jezt, fo rie bas Infefts überf̧aupt, viel grofer uno nusgebiliveter. Die Echale war nun ganj zufanmmengerollt, auf der Dberfladde ligeno, wo fie nad) uno

- nad verwefert iff.

Bon nun an war bas Jufeft feinen Bermanolungen meţr ausgelezt, meil es

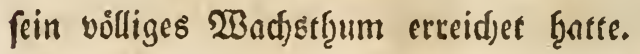

Eine geraume Beit leben dife Jnfeften in foldeem yollfommnen Buftande. Wäf̨reno bifes 
offes Sectraums ift if̧re sebensgefdidnte for. genoe: Bifisueilen, (act)t ober zeţen Sage vor

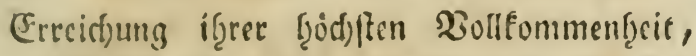
meiffens aber gleidf) in ben erffen Sagen nad, Erercidung oerfelbigen, benn vorber foinnen fie, wegen ber Riurje ifgeer Süfe, nüf bent meift unebuen und geoderigten Slatt nid)t

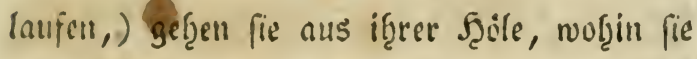

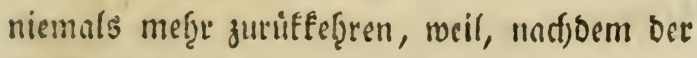
Defel von bell jungen ₹ģierdjen verlaffen iff, Derferbige zufammentollet, bie Mutter in ber faobung noftirbe und verwefet, auth in bem. felbigen feine গantrung mę̧r j" finben iff.

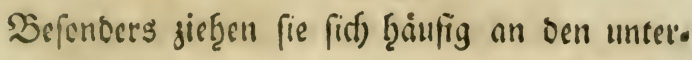
ften Ţęeil ber Slätter, an Denjenigen Srt, wo biefe if̧ren Ltefprung aus ber $\mathfrak{W}$ urzel nef̨. men, weil fie, wegen ber grö(ern waeid)e Diefer Ţ̧eile, bermittelft if̧res Gaugrưfels, 
leichter und tiefer cinbringen, und alfo if̧re Paḩrung beffer finoen foonnen.

Díf gefóief̧et nur einige Seit, in weldfer

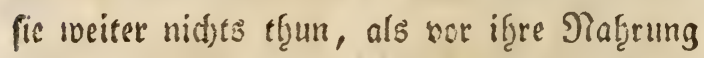
gu forgen, ofine fict) noch gu begatten, ob fie gleid) alle untercinander ḩcrumlaufen. 2on nun an entfefchet eine-widjtige fgaptoerin. berung.

Siff̧er Ȩatten fie groar Fint und wieber Befâffe angeftochen, aber noct, of̧ne in bice felbige eingtoringen, fonbern blok um oen Gaft aus benfelbigen zu jief̧en. Jezt fangen fie an, bie fein gevauten Ţ̧̨eile zu jerfplits tern, fid bis in bie Siefe bes $\mathfrak{B}$ lattes einju= freffen, mad)en in oer Mitten Şólungen, in benen fie eine Beitlang Ģerummandern, und fortlaufen, bis fie fid) wieberum an einem 
Dit Deffelfigen Gerausfreffen, und ob̧ngefeţe nad) neunzef̨n Sagen wicber nuf oce Dber.

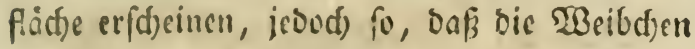
mit ber Dberf̧aut beocfe find. Nun lofige dic Minter bie Dberhaut ab, oricft fie in

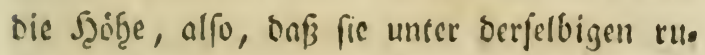
f̧en fan, uno bereitet fidf alfo cine Defe, die nid)ts andet's ift, als eben ber Defel, unter ocm fie felbft einft gebolgren wurbe.

Unter diefen Defel fegt fie fid) jegt, be. fomme fo gleid) eine ountelbraume Farbe, sergnt fict) in die Sreite ans, uno erfdeint num in ber Beftalt einer Sanje. Unterbef. fen Graben fid) aud) bie Männdjen auf sec Dberflad)e berausgefreffen, uns fudben mun, nad) einer Eurzell ふeit, die weiffen Defel nuf, fijen, fo bald fie fie gefunben, nuf bie. felbigen f̧in, und befrudjen, ourd) bie Def.

nung 
nung beffelbigen, bas unter bem Defel li. genbe $\mathfrak{S B}_{\text {eibdibr. }}$

Nad) Der $\mathfrak{B}$ efrudtung laufen die Månn.

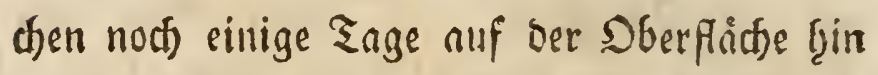
und Gere, ferben denn auf Derfelbigen, uno ifce verweffen Rorper bleiben eine Beitlang auf bem Slatt liegen, bis fie fidf ganj bet. liebren.

Das Weibden legt num oie Fier, und ftirot gleid) Darauf, nadbem fie diefelligen ge. legt f̧at. Gie bleibt nunmegreo too in De. Eel ligen, bis berfelbige zufammenfdrumpft, und fie berunter fâll. 


\section{Ziapitel. \\ (Sharafteriftifd)e Beftreibung \\ Deffelben.}

(S) ie Sigur bes Sunfefts tuberţaupt iff eiformig, ber ganke אơrper mit weifs fen, weidfen, fleinen J̧äirt)en befezt, bie

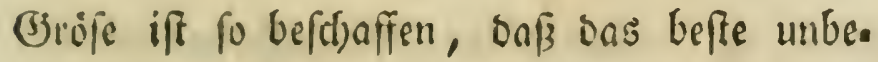
wafuefe Zlug faum cinen guunft erblift. Die Jarbe ift, einige Ţgeile, Die Szaare, bie Juiblthórner, bie Jüffe und bie Babel ausges nonimen, briunfidf. Der gnnze Sörper if weid) und burdficiffig. 2llles biefes ift bei Dem WBeibden, wáf̨rculo feiner grofen Şaupto verinberung, anbers befdaffen. Die broúfe Deffelben ift etwas merfliffer, bie Sarbe wird Dunfelbraun, und das Ganje befommt die Figur einer $\mathfrak{B}$ anje. 
STun betractfte id) joben Theil befonters. खुenn id bas Sgierden von vorne an but(t). forfdie, fo find bie Theildsen beffelbigen fols gende.

I. Der Liopf ift mit sem Srufftut fo genau verciniget, boß fein Llnterfbied

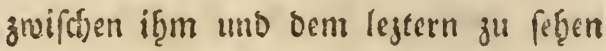
ift. Derfelbige ift balbfugelformig, weidf), mit feinen feaaren befejt, und bzălt ben adjen F̧eil bes ganzen Jn. ffFts. Man unterfábeibet an ifgm fol. genoe Ţęile;

a.) Die 2lugen, bie auf beiben Geiten ein tyalbmal fo weit abftes

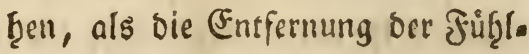

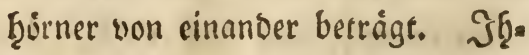
re Sigur gleidgt einer plattgebrif. 


\section{I5}

ten Rugel (Ephaciroioe). Sie figen an ien Geiten bes Ropfs. J̧r Brurd ift ounfelroth, mit etwas fobwargem vermifot, uns if̨re Dúerflåd)e fducinet vielccfigt, wie gefdliffene coclfeine, ju fern.

b.) Die Sulbllobuer erfosemen, in Form eines Gelenfes zufammen. gefezt, uno enoigen fid) in eine burftenformige Epize, oie mit fei. nen weifen f̧ärdsen befezt ift. Eie fins fo lange, als ber Sopf mis Dem Bruftete zufammengenom. men; ooer nod) rinmal fo lang, als bie Babel nm Şinterleib.

J̧̧re farbe ift weißs. Sie be. fetgen aus einer rocidgen Eubfang. 
Eie fino von einer grofen Ent. pfindiddéter, fo, oaf benn bas Junfeft etmas antafter, bie Jjerger fiorner fid) fo gleidy auf beiocm Eeiten zll bewegen anfangen. Sמenn es auf ben Staub ausgegt, fo fino bie Juhtefgorner beftantioing in Şervegung.

c.) Der truno ift Ģier bei biefem Jnfeft fetre fonderbar. Es fiat nidit blop bie Miundpalte, die bie. fer (5)attung yon Sinfeften gemein, und bei diefem Jnfeft fo ourdf. firfitiz ift, als ber librige Rorper. Br if etwas lang geffaltet, um in Sie Ċefäffe einjubringen, uno ţat überbis nod) juei lange fraare, bie auf beiden Geiten ber Mund. fpalte 


\section{$x y$}

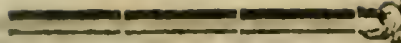

fpalte fervor madfen, aber, fo bald ber Mund erroadfen ift, fids wieber verlief̧ren. Der Muno bat eine sinie in ber Sånge. Die (befptalt ift mef̧re länglid)t, als brcis. $21 \mathrm{~m}$ Sinnoe ift er mit ferte

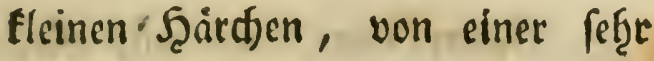
weidjen, meiffen, Durdffidftigen Materie, bewadffen.

In ben exften Sagen, aud faton in ber Beit, wenn bas Jinfeft eine berrad)tlid)e (brö. fe errcidet ţat, fan aud) bas allerbefte be.

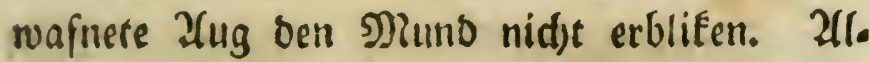
leine, wir foinnen budf nidst an feinem Da. feun żociflen, weil ber Eaugrüfel zur गiaḩ. rung notḩwendig iff.

II. Denjenigen Ţeil, ber zunåd)/t an ben Sopf grånizet, nennen wir bas Zjults $\mathfrak{B}$

ftút. 
ftut. Difes ift bei andern Junfeften oft feţr beutlid) von ben andern ₹̧̨eilen zu unterfheiben; Gier aber ift es fdiwer, meil bas Brufftut mit bem Siumpf uno Ropf in einen Rorper verwartfen iff. Dis if eben bie Urfacte, warum ber

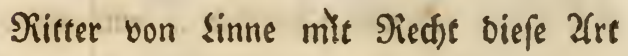
Jnfeften Coccus benennt. $\left({ }^{*}\right)$ Das Bruftitute ift nod) einmal to lange, als Der Ropf, oben runo gewoilbe, unten platt, vori Farbe braun; bie obere Sdja. le, welche bas Infeft bebeft, ift etwas

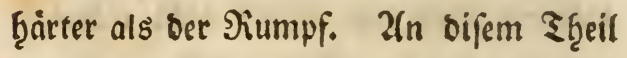
f̧aben wir bie zroeen vorbern füfe zu betradten.

\section{Die}

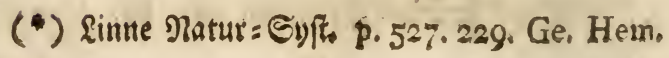


Die zween vordern füffe fint an bem Brufffuif angergeftet. Sie bृaben ben britten Ţzeil bes ganzen Infefts in bets sange. Jore Farbe if meiß, ourd)fidjo tig, uno find von meidjer Eubftang. Sie befteģen:

1.) Zlus ben Schenteln, beten Breio te of̧ngefeģr Den 24 ten Ţ̧eil eines Solles betràgt. Sie fino bzalb fo lange, als bie Fủhltbơrner.

2.) Zlus Dem Echienbein, Difes ift ein Drittheil fo lange, als ber Edjentel, uno um bie J̧álfte bủn. ner.

3.) Xus Dem fuffiblatt. Difes if ein Biertel mal fo lange, als ons Edjienbein, fojmal, uno beftehet aus brei Rlauen (Digitis), movon

$$
\mathfrak{B} 2
$$

bie 
bie zreei oberfte länger fino, als ber untere, uno beren Epize fixt) in etros breite Punften, womit fie fic) an glatten Sberflådjen ţalten foininen, endigen.

III. Der Jinterleib, ober bas britte Gtü Des Sörpers f̧at bie Beftalt eines frump. fen abgefdinittenen Regels. Difer Şin. terleib nimmt an Groófe of̧ngefef̧r ben britten Ţ̧eil bes gangen Jufefts ein; er beftefget aus funf Singen, bie in einnn. Der gefdgoben find. Dife Singe fino fo ftare mit faum merflid)en Şärdjen be: fezt, bafz man fie, auds bei ber beften Bergröferung, faum waḩınef̨men Ean. Sie ţaben ebenfalls die broune Farbe, romit bas ganje Jnfeft bemaḩlet ift. 
Wir bemerfen bei biefem ?̧̧eil,

1.) Die vier legren Jüffe:

a.) Die sneen mittlern, uno

b.) Die zneen lezten. J̧̧ৃe Jigur, Jarbe, Jrojpe :c. ift fajon oben bei bem Brufftúf angejeigt worben.

2.) Nun f̧abell wir nod) bas lezte, nem. lid) bie Babel (Furca), am Ende bes J̧intertheils zu betradteten. Dife Babel beftef̧et aus zroeen ftarfen weifen ourdffidftigen f̧aaren, weldhe ein

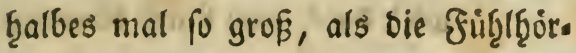
ner fint. Sie fleten offters, wenn Das Jinfeft viber eine füfirge Naterie binuveg lauft, on ben aufferfien Epi. zen an, fo, baßs fid) bas Jinfeft, wenn es ḩerab fallen mơd)te, in etruas ba. mit anţalten fan.

$$
\text { B } 3
$$


3.) Die fünf Ringe am Şintertf̧eil bes Rumpfes, fdieben fid) in einanter, und idf Ģabe bei faneller Bemegung bes Infefts gefeņen, dafi es dife ?Rin. ge rect)felsweife in einander fofiebt uno wiederum ausoef̧net. Eie fint

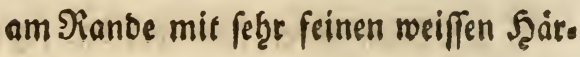
d)en befezt.

4.) Zmifhen bifer (3abel ligt bas $\mathfrak{B e}$. fentlidffe bes 2 Beibdens, nemlid) bie Edjeibe (Vagina). Dife Edreibe mirb burd) zmei fartfe bråunlidje bur(f). fidstige Nebentbeile eingeidloffen, wel. d)e, wenn bas Junfefte geţet, bie Echei. be in bie Mitte zufammen faflieffen, baß man fie faum nocf) erblifet. Die Edxeibe felbft betråge ben britten ₹̧̨eil ber Babel in ber lange. Sie befteţet 
an bem Dbertheil nus zroei ftarfen Epizen, bic, wenn die Befrudtetung gefdief̧et, fict) iffnen, nad) gefdeţ̨e. ner $\mathfrak{B}$ efrudstung aber wieber etwas náţer brifammen fref̧en. 2fus benen obenbefdriebenen Tebenţ̧eilen Der Edreibe entipringen an benen auffer. ften Ţ̧ৃeilen berfelben bie zroei ftarfe Şaare, weldje die Gabel formiren.

Das $\mathfrak{B e i b d j e n ~ u n t e r ( d ) e i b e t ~ f i d ) ~ n u r ~ b u r d f ~}$ bie etroas merflidfere Bròfe, burd) bie bem Weibden wefentlide Ģeburtstheile, unb, gue Seit feiner $\mathfrak{B e r w a n b l u n g , ~ D u r d ) ~ b i e ~ o b e n ~ a n a ~}$ gejeigte Berainberung feiner (Beftalt.

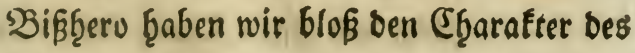
₹̧̧ierchens überḩaupt gefdjilbert. Jezt verfu. den wir, feine $\mathfrak{B}$ erridftungen zu Durdffpąten.

$$
\mathfrak{B}_{4} \text { כei }
$$


Bei jebem organifden $\mathfrak{B e f e n}$ bat man vorzugglich) vier groffe $\mathfrak{B i r f u n g e n ~ z u ~ b e m e r t e n : ~}$

1. Das. Wactsthum. Difes haben wir fthon oben, vom Ei an bis zu feiner $\mathfrak{B}$ oll. fommenţeit, gefdjilbert, uno betradten bier nur

a.) Die Materie; aus weldjer es wåd) Bt, und fid) bis an feinen Sod erţålt. Dife. ift ein feiner, füf fer, mit etwas Gnurem vermilds: ter Gaft, der bei einer jeben 2(rt won Znanasplanzen unterfaieden iff. Sei ber milden 2lnanas ţat bie Săure bie Dberţano, unb über. freigt bie füffen ₹̧̧eile weit. 2lus ber Exfab̨rung wiffen wir : Dnß biefe Jnfeften auf ben wilben 2Ina. naffen, 
naffen, in 2tnfergung if̧rer 2 er. meţrung, viel ftarter unto in grơf ferer Mienge borf̧anten find, als auf benen zaf̧men ober orbinaren. Die Coccus muß alfo die Enure meb̨r lieben, als ons füffe $\mathfrak{B e}$ en, rocil jene den Rorter wor ber Jåufniß̧ beffer verwab̨ret.

Dife Naterie erf̧âlt $\mathfrak{E B}$,

b.) vermittelft einer thiunorpalte ober Eaugruifels, Der, in 2anfef2ung Des ganzen Rơrpers, flein, und fo eingeridgtet ift, bas bas Infett ben Gaft aus ben inner. ften Ţ̧eilen bes Blattes bequem Jeraus faugen, uno firt) erb̧alten fan. allein, biefe Munofpalte ift fo zart, bak Das Jnfeft, aus bert

$$
\text { B } 5 \text { நृärtern }
$$


Ģärtern Tḩ̨eilen bes Blattes, Den Eaft nidjt ţerauszuzieţen per: mag.

c.) Difem Fef̧ler நृat aber bie Natur fijon nbgeţolfen, uno bas Infeft an einen andern Drt gerwiefen, roo bie Dberflädse um einen groffen Brab weid,̧er ift, uno bie Ş̧eil. d)en noch reţr blaß ausfeţen. Das Jnfeft f̧ålt fic) nemlid) am liebften an bem Urfprung ber Pflan. ze auf. Der Saft, ber an bifem Ort fid findet, ift gerabe ber befte. Er ift b̧ier nidje in bem bुob̧en Brabe ber Feinf̧eit filtrirt, uno bis in bie geiftig: Theild)en abge. fondert; benn bife Materie waire villeid)t zu fकjarf, und fönnte if̧ren 
fo fein gebnueten Siorper leidft zer. ftoren, (wie bei bem Mittel wiber bife Jinfeften ein Beifpiel ange. fübret ift). Der Drt, no die Iill. feften if̧re গaţrung ant beften fin. ben, bat nod) meţrere Bequem. liajfeiten, die if̧in ebenfalls bem. relbigen reizento madfen. Şier fan weber bie Eonne, nod) viele an. bere $\mathfrak{W e f e n , ~ b i e ~ b i f e n ~ J u f e f t e n ~}$ Echaben zufügen fönnten, if̧nen jufommen; fie bुakan alfo vor bes nen meiften sufferlidfen 2(nfällen bier Ed)us.

Die 2trt, wie es nun die Naţ. rung an biefem Drt ourd) feine $\$$ n. frrumenten ergृailt, ift bife: 
d.) Suerft löfet es bie Dberf̧aut $a b$, und bereitet fid) cine Defnung bis auf bie Scaut bifes (Berwebes, in welcjem die Brefäffe fef̧r fein zus

' fammen geflodten fino. Es zer. reiffet bife Sheildben, und bei ber geringften Berlegung einiger Bes fäfe fleffet fogleich ber Saft wel. renformig ḩeraus, quillet bem In. feft entgegen, uno alfo ţat es einen Borrath von Saft. Es begnuget fict) aber nicht an bifér Defnung, bie es gemactst f̧at; es zerfplittert bie Sheildsen in einem grofen $4 \mathrm{~m}=$ fang, uno fauget alfo baburd) im. mer neue Raterien. Şierourd aber wird das S3latt ganz verbor. ben, ber Umlauf des Eaftes in bifer 
Difer Gegend leibet Edjnden, bie Bervegung beffelben, in benen una ter ben Gefäffen ligenden Ţ̧eil. d)en, wirb geţemmet, bie feine Ja. fern, (roeld)e jufammen genommen Den Baul bes Befáfes oder bes Sanals, worinn fid) ber Gaft be: weget, ausmadjen, ) werben fafts loß̧, aller গaf̧rung, zur Jorffezung uno Erf̧altung if̧res Saues, be. raubet, immer untuidtiger, uno ber gainglidjen $\mathfrak{B}$ erwefung ausge. fezt, weldse audf) in furzem barauf erfolget. Eben bab̧er entftȩृet aud) bie $\mathfrak{B}$ er fdibenţeit Der braunen Jlefen.

II. Wie fid, bifes Infette ernåđ̧re, ţa. ben wir fdjon oben ùberб̧aupt, gefage. 
Wir wollen es nun genauer betrad.

\section{ten:}

1.) Das Jnftrument, ons zur Zne f(f)affung Der গią̧rung oienet, ift ser Gaugrufel.

2.) Die Gelegenţeit, die if̨m die Patur an die J̧and gibt, ift ber 2lufenthalt in Dem Slatt, in oem Det ఇaţrungsfaft entḩalten, uno weld)es zur Nab̧rung forouţl, als aud) zur Befáchung und fortpflan. zung beffelben, gleid) gefdrift ift.

3.) Die 2trt, wie es fict) náb̨ret, ift folgende:

\section{Wenn sifes Jnfett nusläufet,} fo reiffet es fier uns on auf ber Sberfládje des Slatts ein SBefäß, vermittelft feines Snugrũfels, auf, 
um ben Eaft Daraus zu faugen. शach bifem, menn es auf ber Dberflàd)e menige गlaḩrung findet, bringet es fid bis in bas Jnnerfte Des Blattes, zerfplittert aud) Ģier bie fein gebauten Giefáffe, bamit if̧m ber Saft entgegen quille, uno faugt alsbenn mit frinem Saug. riffel ben etwas fauren Saft ber PPlanze, aus. a(llein, aud) jeje mußs bas Infeft nod) einmal ben Drt verlaffen, um feine Naf̧rung 3u fudfen. Es gethet nun meiter fort, und madot gleidf fam f̧ölun. gen, bie ben Gruno zur Entffe. f̧ung ber braunen, flefen, ent. bzalten. 2(ud) b̧ier begniget es fich nod) nirfse, fonbern reiffet mit fei. 


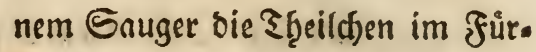
ficigebcen immer weiter auseinan. Ser, bis es balb mef̧r gegen bie Sberfiäche des Slattes fonmt, zu verfdibenen Beiten aber wieber in sie Tiefe zurü geb̨et, umo jene

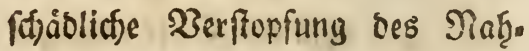
rungsfaftes verurfactet, reldje bie Begeno, wo bas Jufeft um fict) gefreffen, bals in Fåulniß̧ bringet.

भiBenn sas Gnfeft Die unterfte Befäfle aukgefogen bुat, fo fonmt bie 3eit, tro es fid gegen bie Dberflàd)e des $\mathfrak{B l a t t e s}$ nåţert, und fid wieber Gerausfriff. Wenn bas Weibøen, nad) oem Uusfreffen, ifrren Defel gubereitet bुat, fo nimmt fie maf̧rfabeinlider $\mathfrak{B}$ eife feine

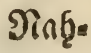


Siag̨rung meţ̧ ju fid). Eie fdwillt also, in ber Bieftalt einer Nonnje, auf, uno figt feft unter bem Drfel, wo zwifhen ber Coccus unt bem $\mathfrak{B l a t t}$ die Eier ge. funden werben.

Das Mainndjen ţat fidf) jegt aud) ausgefreffen. Dod) ift es

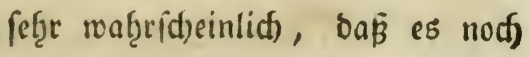
Siaf̨rung ju fid) neţme; benn (b)e. legenţeit uno Beit b̧at es genug, Difen if̧m fo angeneţmen Saft in fici) ju zief̨en.

III. Dife Coccus ţat eine eigene 2frt, wie

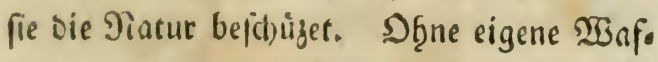

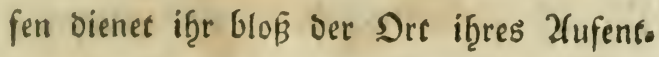
Eqalts zur Befduizung. Eis woţnet nem. lid), fo lange fis bie (Eier leget, unt in ber 


\section{4}

erften Beit iţrer Bieburt, unter einem De. Fel, ber fid) fonften bei Eeiner andern 2(rt Der Coccus - Infeften finoet.

Difer Defel fobuget das Jnfeft fur פBillo uno Regen, borzuglich aber für ocm

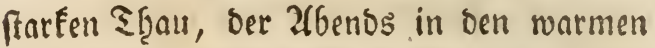
(J)genden, fo roob̨l auf ber norblicten Ril. fte von 2ffrifa, als auf ben 2(ntillifaen Jil. feln, uno ḩauptåd)liç in Zmerifa, auf bie Pflanze fället. 2luffer bifer Seit fino fie ourd) if̧ren 2lufentţalt, ber allemal jur beften Bcit geroaifelet wiro, in bem Inner. ften bes Blattes, uno meiftens am Ur. fprung beffelbigen, befaruzet.

IV. Die Begattung f̧at in Sem ganjen Jit. feftenreid) viel aufferorbentliches. Die (3efdidfe berfelben ift fdjon oben ergifilet worien. 
bourben. Dod) mollen wir biefelbige Ģier weiter ausjuforfdien fudfen.

A. Die ăuffere Umftande, weldje bie Эla. tur barbieter, find folgende:

Der Srt ift Der Defel. Difer De. fel ift meiter nictits, als ein aufgerif. fener Theil ber Dberf̨aue Des Slatts, unter bem alfo bas Jnfeft befduijet werben fan. Er ift weiplich), zirkels formin, an ben meiften Drten unourdf. fichtig, aus einigen Deffnumgen befte. beno, ziemlid) grob, uno nad) allen Sheilen gefdift, ons Jnfett ju bede. fen, uno zu beid)üzen.

Eeine Entfteţung ift rab̨rfadeinli. d)er Weife folgende:

Er fdeinet eigentliff berjenige ?heil Der Dberb̧aut zu renn, Den das in ben (5. 2 innern 
innern Befåffen des Slatts Gૃerumwan. bernde Jnfeft, vermog feines গatur. triebs, bauet, uno burd) bie 2(rt ber Bervegung, die es machet, zu berje. nigen form bilbet, bie wir wirflid) am Defel wabernef̧men. Dife হeis. nung roiro um fo ronţrfdeinlidber,

a.) weil bie Dberf̧aut aufferft leidfe abgeriffen werben fan.

b.) Ueberoif fommt oer Defel, mit Den ribrigen SGeilen ber Sberţout, fo feber in sen Seftanotgerilen bes nezformigen Gervebes ůberein, onß, wenn fo moht ber Defel, als jence abgeriffene Sheil ber Dberţaut, un= tet bem zufammengefegten Werg!e: ferungsglas betradjet wiro, man faft Eeinen Unterffoies gewaf̧r wiro.

B. 
B. Die SiBstfjenge find fajon oben ange. jeiget moroen.

C. Die Nisibrten, bie vermutf̧lid) in Dem Jnnern bes ßlattes féme Tlaf̧rung mef̧r finben, fo wie bie Mländfen, tretten jejt wieder jur Dberfläd) Ȩer. ai1, uno burthreiffen alfo bie Dberf̧aut. Die lejte flię̧en jezt vom Sort weg, no fie fid) aufgetralten. Die erftern bilben fiif) bamit ben Defel auf bie obenbe. fdriebene 2(rt, woju fie vermutb̨lich) if̨. re Emppindidsfeit gegen bie Suft, if̧re Epeife, uno if̧r Naturtrieb, reizet.

So balb oce Defel fertig ift, reget

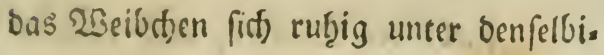
gen fiin, und beginnet nun ju erwact). fen und fich) ju verwanden. Este aber nod) bie Berwanotung vor fid) geţet,

$$
\text { (c) } 3 \text { fornmt }
$$


fommt bas פRanndben, fejet fich auf bie Mitte bes mit einer Defnung ver. fef̧enen Defels, befructet das $\mathfrak{B B}_{\mathrm{B}} \mathrm{ib}$. den, gleid) nad) ber ßefrudstung ges het es weg, uno firbt. Das నrcib. d)en wiro nun groffer, verwandelt fich, uno erfácinet nun in oer Beftalt einer Wanze. Nun fofeinet es ganz of̧ne Empfinoung uno Beroegung zu fenn, und felblt feine Bewegung zu füf̨len; uno in bifem Suftand legt es bie Eier. So bald bie Eier gelegt, und fo ers madjfen find, bag fie fdjon of̧ue bie Mutter fid felbft erţalten fơnnen, fo frirbt bie खutter, uno verwefer.

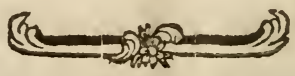




\section{Ziapitel. \\ Mittel Dagegen.}

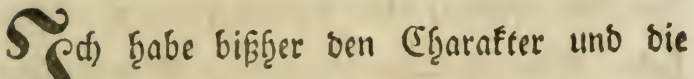
(3)efdidfte bes 2(nanasfdjilbs abgemaha.

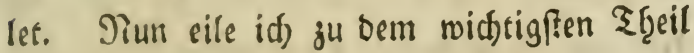
meiner Ulnterfudjung, zur Setradftung iૃ̨res Edjabens, uno ber $\mathfrak{Z}$ trt, fie zu vertreíben.

Der Edfaben bes Junfetts befteţe eigento lid) Darinn: Dás es bie Befáffe aufreiffet, um bie Tinģrung baraus ju faugen, und fiid) bar. inn oufzuţalten. F̧ierburd) entffetęen, thęeils

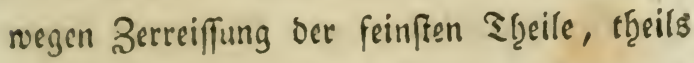
wegen Beraubung des Naf̧rungsfaftes, zuerff braune flefen, uno balo barnuf cine Şåulniß̧, bie fidf) juerft an sinent beftimmten Drt anfe. zet, aber balo burdi bas ganje Blaft fortpfian. 
zet. Sefonders daib̨net fie fid) von bem ver. lezten Srt bes Bintts an, bis an Den Urfprung Deffelbigen, aus, woferbfien, wegen der 2 bee de bifer Şreile, eine folnelle Fåulnif vorge. bet. Bon ba aus oringet bie Berwuiftung Dergeftalten bis aufs Sterz Der Pflanje, bis fein einiger gef̣unter Ş̧eil meţr bavon vor. handen ift, und biefelbe oft von feloften, ober bei ber leid)teften SBerifitrung, auscinander fället.

Man glaubte anfänglich) nid)t, baßß bifer Echaben fo grof fene; nadbero aber erfuf̧ren es biejenigen, weld)e dife Pflanze in Mienge in if̧ren Blasţåufern uno Błlastâften freţen bzatten. Nan mad)te baf̧er auf alle móglicte 2(rt $\mathfrak{B}$ erfuche), dife siufe ju vertreiben; bis jezo nber fonnte man nod) fein braudbares Mittel bagegen ausfündig madjen, weil bas wibcr 


\section{$4 \mathrm{I}$}

wiber sife Planjenfióberer gebrautbte (Gift, immer and) cin Bift wioce Dic Yflanje feloft, war.

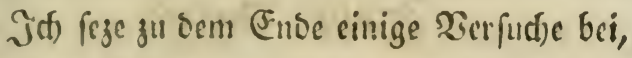
savon bie orei erftern nid)t wirffam genug, oder Sod) mit verfdjiornen foflimmen Llmftän. ben verbumben waren, ber lejte aber meifens mit ocm beften Exfolg gefronet rourde.

\section{Eerfer Werfitch.}

Eoldser beftunte in foigendem: Man jer. friez Edmefel, fo Elein als immer moinglid) war, uno befremete die Pfanjenbláter oa. mit. Difes Mittel bat aber bie geţofte $\mathfrak{B i t}$ fung nid)t gettran, weil ber Estwefel nid)t in das Innerfte oer Dlatser, nofelbften fid) bod bie zu toosnoc jnfeften aufg̨alten, cinoringet. Silleidgt baitte er aber bod getuirfet, wenn

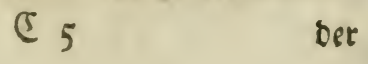


Der Edroefelftallb nidfe, bei Segieffung oer Z(namaffen, wåre abgewafdjen worben.

\section{כwoiter $\mathcal{D}$ erfuch.}

Man ma(t)te mit bem Gdjruefel noct) einen anbern Derfuch), nur mit Dem Unterfdieo, Daß man if̧n anjuำete.

פan fufce nemlid) mit oempelbigen immer auf ber Dberflictie ber Slätter alfo fiin und her, baßz ber Dampf Davon, vermittelft feiner Feinfeeit, in sie innerfte Theile berfelben orin. gen follte.

Difer Berfud ffien anfänglich einen glü. lidjen Erfolg zu ţaben; benn es blieben dife Jinfeften einige Beit vollig aus, uno man fputhrte niđts meţr von benfelben. Siad) bૃer aber fanden fie fid) wieberum in eben ber פen. ge éin, wie zubor. Die Lurfadje, warum fie 
fid) anfänglich) verlof̧ren, lino alsoenn wieber bervorgefommen, fdjeinet onrinn ju ligen: baf bicjenigen, die fdon theils unter if̧rem Defel bृervorgeloffen, theeils nod) Darunter bet. borgen gewefen, leidtlid) von bem Edynefel. sampf founten getoost weroen; biejenigen nber, sie im Snnerften ber Blinter verborgen lagen, rrfufgren difes Edjifjal nid)t, weil ber Dampf in einer folden furgen 3eit oas Junerfte ber Släter nidft ourdjoringen fonnte. Eie fa, men alfo, nads einiger Zeit, wieser, ober fro. d)en vidmetre nus benen innerften auf bie älf. fere Sfgeile oer Blåtter, legten Eier, uno pflanj. ten ifge (Jefdoledt auf bas neue fort.

\section{Dritter Derfuch.}

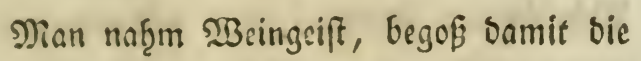
Pfinnje fo frark, Daßz fie bsi nahge felbft bavon jer, 


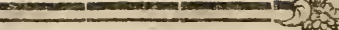

zerfreflen rourde, und fand wirflicf), daßj-ber झुsingeift alle biejenigen Jnfeften, bie fidf alif Der Dberflátje der Balatter befanden, ge. tobet b̨atte. Die 2 Baḩrtecit bifes Erfolges be. fiátiget fich noch liberbif burct) eine anoere von mir gemadate Probe. Jaf) fano nemlid), nad). Dem id liber mef̧rere Jnfeften etroas SBein. geift gegoffen fratte, baß fie in einer Minute tos waren. Jnoeffen fafee idy ood) bald, saß Der $\mathfrak{B e i n g e i f t ~ b i f e ~ J n f e t t e n ~ n i c h t ~ a l l e ~ g e t o ̈ b e t ~}$ batte; Denn nad) einiger Seit Eamen bie Jn. feften bृåufig wieser hervor, um ber feḩr be. greificfen Ltrfad)e willen, weil serfelbe nidje bis in die Tiefe ig̣ter innern $\mathfrak{B} 0$ ḩnungen ges brungen war.

zlle sife Berfucbe erfüllen nod) nidjt die 23: nicts befriebigens genug.

Ran 
Dian forfdete alfo weiter nad), uno fiel enolid) auf folgenden ganj branchbar erfunde. nen Berfuct), Der, nact) viclen angeftellen Bes. obad)tungen, felten feţl gefdlagen f̧at.

Man fejte hicbri voraus: baf es notḩwent. big feve, bie Dfanzen aus ber Erbe hacrnus zu nef̧men, weil fonften feine Materie in bie, Durd) biefrlbe bebefte ?̧̧eild)en ber Planjen, einoringen fonnte, uno fam baburd) allf folo gendes zuverláfige ఇaittel.

\section{Dierter Derfuch.}

Man bृebt nemlid, die Pfanzen, wie bea reits erwefgnet worden, aus ber Eroen ந̨ernus, uno lafft fie zivei nud) orei sage lang frei in bem 2(nanasţauß ligen, woourd) fie ein we. nig roelf roerben, und fid) alfo fierourd) ifcre

\section{Eafte,}




\section{6}

Eafte, die rigentlidge Dinţrung bes Jnfefts, in etras verlieren uns ausbinften.

Difes abwelfen ift fo nothig, saß, wenn bie Pflange, gleid) uach bem 2 (ustereben, in sie nad)fetgente sauge foume, die Jnfeften fogleid) roieber vorbzanoen fino, invem folde, wegen bem vielen Eaft berfiben, nod) bie Tiaf̧rung wie guvor finden.

BBenn fie auf befagte Irte ein wenig toelf geworien, wiro ein groffes Biefáb oder Suber (Rufe) mit Waffer angefullet; fodann reerben 24 Etunoen lang Tabaffengel (*) Darinn eill. geweid)et, bis Das $\mathfrak{W a f f e r}$ gang braun bavon mirs, uns, fo viel es immer mogglid), ben Eaft bes 'sabaks ausnelogen hat. Tiad) bi. fem wiro bet Sabak mit benen Şånden ausge. orufet,

(*) Nicotiana ruftica, panicula \&c. Limm。 Syyt. Pant. 
Druffet, bie Syflanje in difes $\mathfrak{B}$ nffer getţan, ebenfalls 24 Eenmoen barinn gelaffen, nact) 3erfluß bifer Deit wieber ţeraus genommen, in einem friffien faubern $\mathfrak{B a f f e r}$ ab , und aus. gewafden, uno heernact)mals einige Sage ver. Esf̧rt aufgef̧ainget, bamit forwof̧l bas reine als unreine $\mathfrak{B} a$ ffic ablaufen und villig abtripfeln fan. Enolid) wirt fold)e, wie gerosignnlids, niederum in bas sof̨beet verpfonjet.

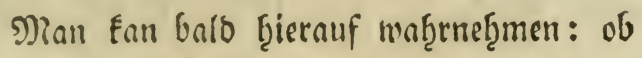

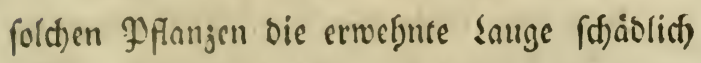
ober unfdablid gewefen? inbem fie langftens in ad)t bis zeţen Eagen anwad) fen und aufa red)t fref̧en, ober umfallen uno verberben wer. ben.

Tiun will id) nur nod) jum $\mathfrak{B} e$ (d)luß cini. ge zufállige 2fnmerfungen Ģierüber beibringen. 
Die wilbe voer faure Ifnanaffe (*) fonnen bie Saize oder bie sauge viel weniger ertra. gen, uno fę̣en baf̧er übler nach berfelben aus, als bie orbinairen ober zaţmen. (**)

Eo viel

(*) SBilbe 2tnmas, (Bromelia Karatas) mit nufred) frelyensen Silattern, uno gethiuften fetfijencen S3lumen. Nhexocotl. Moris. hift. 2. f. 4. T. 22. f. ?

Bromelia Karatas, acaulis, floribus aggregatis felfilibus fubradicaulibus. $\mathscr{J} a c$. Americ. 19.

Bromeia, foliis fpinofis oblique recurvis, fructibus dispermis, racemofis. Hort. Upjal. 73 .

Karatas, foliis altiffimis, anguftiffimis \& aculeatis. Plum. gen. 10? Dab mittingige 2tmerifa ift Deffen Jomath. 4

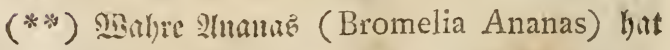
gefrum; = jad)lidbe, in eine Exize fich ensis

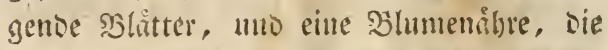
mit cinem 3opi gejieret iff. Tiew. Ehret. T. 2.

Bro- 
viel wir aus ber Erfab̨rung wiffen, fornnen bie

olgne

Bromelia Ananas, foliis ciliato-fpinofis, mucronatis, fpica comofa.

Bromelia, foliis fpinotis, fructibus connatis, caulem cingentibus. Hort. Cliff. I27. Hort. Upfal. 73 .

Ananas aculcatus, fructu ovato, carne albida. Trex. Ehret. T.2. Dije waljre 2 Inanaz bat folgente 2tbaniorungen:

a.) Zuterbut, mit furnmibenfurmiger frudst uto gellocu fleijd)e. Ananas aculeatus, fructu pyramidato, carne aurea, Tournef. inft. 653 .

6.) Ëtadblid)e Stnanns, mit Égelpurmiger Fritclt uno getben Slciftce. Ananas aculeatus, fructu conico, carne aurea. Pluk. Spec. 20.

r.) Sinnig?apiel mit bellgrumen Slattern, weldye an Dcm Panie fuum cinige Cinges: einichnitte haketr. Ananas aculeatus lncide virens, folio vix ferrato. Dill: Hort. Elth. 25. T. 21. f. 22. WBid) Ët in Reufinien แ⿰ Gurinam. 4 
obgne Stadjeln die Zauge gar nidjt auş̧alten, fo, dak fie, bie grơften nicht ausgenommen, entweber ganj verwelfen, ober fich mit Niütze, und in einer fef̧e fdolimmen Beftalt, erţalten. 2(ud) mit bem Degieffen berfelben muß man fich) in nơft nef̨men, bis man glaubet, daß̧ fie ciritge $\mathfrak{B} u r z e l n$ gemacht haben. Sobalo man fold)es merfet, fo fönnen fie, fo moḩl unten im Topf mit oer Siọtre, als aud) oben mit sem Giefpepf, befprenget werben.

Sollte ficf) bie uno da cine anonas finden, weldje fajon Jriditen angerezet f̧at, fo pafit suct) in bifem fall bas Mittel vollfommen. Denu man ḩat die Erfaḩrung, daß̧ fruictten, roeldae fith theils erft zeigten, theils aud) (d)on fo großz wie ein Ei waren, dife Befgariolung bennoch ausgeftanden ḩaben, uno zu if̧rer Siei. fe gefommen fino. Difer $\mathfrak{B e r f u c h}$ bet einen 
fo guten Erfolg getrabt, bonf fid) Dife Jufeften billig verlog̨ren Ģaben.

Uleberf̧aupt bin id für ben ridjtigen E. folg bes ganjen Berfudjs $\mathfrak{B u r g e , ~ w e n n ~ n u e ~}$ meine Borfdrift genau onbei befolget wirb.

\section{2)}

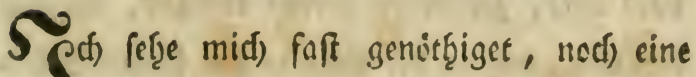
- furze $\mathfrak{B}$ effreibung von anbern. Infefter bier anjuhąangen, bie vorz̧niglidf, mit oer Bromelia, auf ber 2(nanas fidf) auff̧alten. Es fino foldse bie Coccus Hefperidum uno ber Papilio Dido. Jid) fdjilbere fie aber nur ganj furz, weil fie beebe bereits bon Limme befdrieben morben finto.

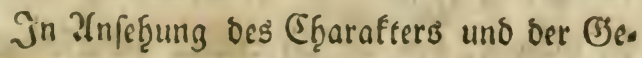
fdidfte bes erffern Snfefts, berufe idf mids

D 2 gång. 
gånjlic) auf bie Sefdreibung bes Riitters von Linne, und reze nur nod) eine 2(nmerfung Gief̧er, bie idb) bafelbft nicht gefunden ḩabe. Difes Infeft nemlich unterfderibet fid) von ber Coccus Bromelia Darinnen: Daß̧ es fich bei weitem nicft fo ḩåufig, als jenes, finoet, baß es überdiß auf ber ganzen Pflanze, balb ba, balb bort, zerftreuet bृerum friectet, vorzüg. lid) aber, dą̧ es der Pflanje gar feinen Edja. Den ţ̧ut. Das legtere ţabe id) an einzelen Pflanzen verfucts, uno immer beftátiget ge. funden.

Die Urfacte sifer Erfdjeinungen ift fef̧e Flar. Denn oa fie nur felten ein Befäaz zer. ftòret, fonbern nur ouf ber Dberfläd)e beer. um geţet, fo fället ber Scauptfdroben bei bi. fer binneg, den bie Bromelia verurfactet. 
Bei bem Papilio Dido (*) fege id, auffer bem, was Liune gefagt, nod) biinzu: 3 enn bie Siaupe fid) in bie Suppe verivan. belt, fo fpinuet fie fid vorţer zwifdsen zwei Slattern ein, uno bleibet fo lange darinnen, bis ber Ed)metterling f̧erbor fommt. Di. fer Papilio wirb aud) auf andern ame. rif́anif̧̧en SPfanjen ange. troffen.

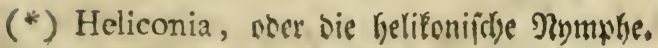
Lim.

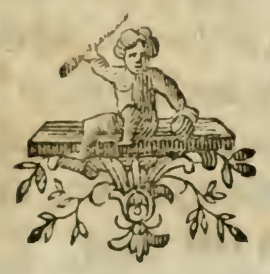

D 3 


\section{Ẽrflårung \\ Der \\ Ruffertafer.}

A. Die Coccus Bromelia, yom Ei an, biş fie nusgewachjen ift.

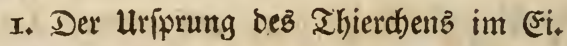

2. Das fibon etwas erwadjiene (Fi, nebft ber anhångenoen Şaut.

3. Das Ei, woran man ichon bie Ringe er= blif́et.

21nmertung. N.I.2.3. fonmen mit blo= fen 2lugen uidht gefeljen werben.

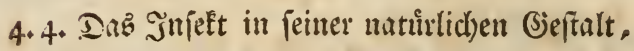
mit sen Firblhornem uno (jabel, unt zwar

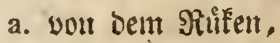

b. yon bem Sauch anjuieken.

5. 5. Das Snjett, f(d)on villig erwad) (en, nemlid)

a. won bem Saudb),

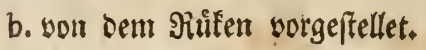

c. Di= 


\section{6}

i. Cind bic Fleten, die man in ben Biefiffen Dez Şlitts, wenn fie abgefturben fmo, ans trift. Sie find and fort ant Ende ber Defel ju febert.

k. Dab Rieibchen, bejombers aligcbilset, wet: cheš, went es bie Eier gelegt hat, aljobalo ftirtset. (Sileicl) falls 3200 mal nach bem Diat ineter sergrifert.

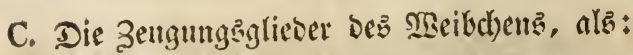

a. Die Gibeide (vagina) an bem Spintertheil Deb Riviper.

b. Die juet Retentheile, weld)e sic Edyeise einjchlieffen. Eben bije Iheile werben hiet nod) genauer zergliebert, uno zwar:

a. Die Giheibe alfo erweitert, saf́ bas Mainud)en, mit feinem friemenfirs: migen zengungsglies, Kequem bie $216=$ fid)t Der פatur ausfiflyren fan.

\&. Die Pebentheile, an Deren Enoe swet frarke Jaane befindlich fints, aus wel: d)en bie Babel ifyen Hriprnng nimmt, gleid)falls ermeitert.

\section{Stuttgart}

georuft tei Sohann Jhbilipp Erharo. 







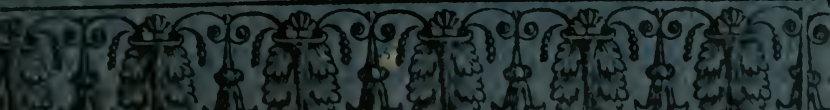

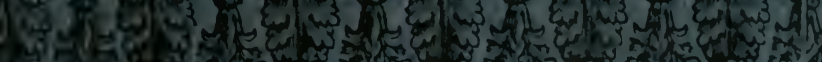

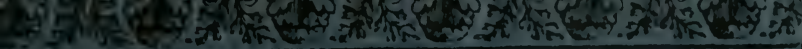

n.

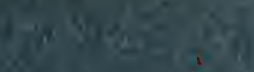

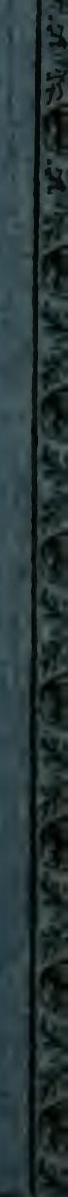


